\title{
Asymptotically Antiperiodic Solutions for a Nonlinear Differential Equation with Piecewise Constant Argument in a Banach Space*
}

\author{
William Dimbour#, Vincent Valmorin ${ }^{2}$ \\ ${ }^{1}$ UMR Espace-Dev Université de Guyane, Cayenne, Guyane \\ ${ }^{2}$ Laboratoire C.E.R.E.G.M.I.A. Université des Antilles, Pointe-à-Pitre, Guadeloupe \\ Email: "William.Dimbour@espe-guyane.fr, Vincent.Valmorin@univ-ag.fr
}

How to cite this paper: Dimbour, W. and Valmorin, V. (2016) Asymptotically Antiperiodic Solutions for a Nonlinear Differential Equation with Piecewise Constant Argument in a Banach Space. Applied Mathematics, 7, 1726-1733.

http://dx.doi.org/10.4236/am.2016.715145

Received: July 25, 2016

Accepted: September 13, 2016

Published: September 16, 2016

Copyright $\odot 2016$ by authors and Scientific Research Publishing Inc. This work is licensed under the Creative

Commons Attribution International

License (CC BY 4.0).

http://creativecommons.org/licenses/by/4.0/

\begin{abstract}
In this paper, we give sufficient conditions for the existence and uniqueness of asymptotically $\omega$-antiperiodic solutions for a nonlinear differential equation with piecewise constant argument in a Banach space when $\omega$ is an integer. This is done using the Banach fixed point theorem. An example involving the heat operator is discussed as an illustration of the theory.
\end{abstract}

\section{Keywords}

Asymptotically $\omega$-Antiperiodic Functions, Differential Equations with Piecewise Constant Argument, Semi-Group

\section{Introduction}

We are concerned with the differential Cauchy problem with piecewise constant argument:

$$
\left\{\begin{array}{l}
x^{\prime}(t)=A x(t)+A_{0} x([t])+g(t, x([t])) \\
x(0)=c_{0}
\end{array}\right.
$$

where $A_{0}$ is a bounded linear operator, [.] is the largest integer function, $g$ is a continuous function on $\mathbb{R}^{+} \times \mathbb{X}$ and $A$ is the infinitesimal generator of an exponentially semigroup $T(t), t \geq 0$ acting on the Banach space $\mathbb{X}$. The main purpose of this work is to study, for the first time, the existence and the uniqueness of asymptotically $\omega$-antiperiodic solutions to (1) when $\omega$ is an integer.

Differential equations with piecewise constant argument (EPCA) have the structure “2010 Mathematics Subject Classification: 34K05; 34A12; 34A40. 
of continuous dynamical systems in intervals of constant length. Therefore they combine the properties of both differential and difference equations. They are used to model problems in biology, economy and in many other fields (see [1]-[7]).

The study of the existence and uniqueness of periodic solutions of differential equations is a well-established fact. The concept of asymptotical periodicity has been introduced to handle phenomena which behave periodically as time grows (see for instance [8]-[10]). However, antiperiodicity has a great importance in the qualitative study of differential equations. For instance, many phenomena in biology, ecology, quantum physics and engineering are antiperiodic (see [10]-[17] and references therein).

Recently, the authors of [18] introduced the concept of asymptotically antiperiodic functions and studied semilinear integrodifferential equations in this framework. In [19], a new composition theorem for asymptotically antiperiodic functions is proved. This result is used to show the existence and the uniqueness of asymptotically antiperiodic mild solution to some fractional functional integro-differential equations in a Banach space. Motivated by [18] and [19], we will show the existence and uniqueness of asymptotically antiperiodic mild solution for (1).

This work is organized as follows. In Section 2, we recall some fundamental properties of asymptotically antiperiodic functions. Section 3 is devoted to our main results. We illustrate our main result in Section 4, dealing with the existence and the uniqueness of asymptotically antiperiodic solution for a partial differential equation.

\section{Preliminaries}

Let $\mathbb{X}$ be a Banach space. The space $B C\left(\mathbb{R}^{+}, \mathbb{X}\right)$ of the continuous bounded functions from $\mathbb{R}^{+}$into $\mathbb{X}$, endowed with the norm $\|f\|_{\infty}:=\sup _{t \geq 0}\|f(t)\|$, is a Banach space. The Banach subspace of functions $f$ such that $\lim _{t \rightarrow \infty} f(t)=0$ is denoted by $C_{0}\left(\mathbb{R}^{+}, \mathbb{X}\right)$. A positive number $\omega$ being given, $P_{\omega}(\mathbb{X})$ will be the subset of $B C\left(\mathbb{R}^{+}, \mathbb{X}\right)$ constituted of all $\omega$-periodic functions; it is also a Banach space. We recall the following properties of antiperiodic and asymptotically antiperiodic functions. We refer to [18] where they are proved.

Definition 2.1. A function $f \in B C(\mathbb{R}, \mathbb{X})$ is said to be $\omega$-antiperiodic (or simply antiperiodic) if there exists $\omega>0$ such that $f(t+\omega)=-f(t)$ for all $t \in \mathbb{R}$. The least such $\omega$ will be called the antiperiod of $f$.

We will denote by $P_{\text {மap }}(\mathbb{X})$, the space of all $\omega$-antiperiodic functions $\mathbb{R} \rightarrow \mathbb{X}$.

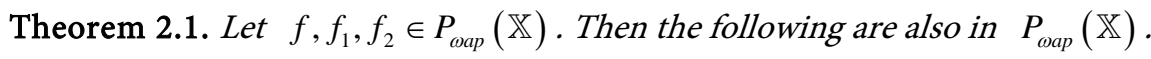

i) $f_{1}+f_{2}, c f, c$ is an arbitrary real number.

ii) $g(t):=\frac{1}{f}(t)$, provided $f \neq 0$ on $\mathbb{R}$. Here $\mathbb{X}=\mathbb{R}$.

iii) $f_{a}(t):=f(t+a)$, $a$ is an arbitrary real number.

Theorem 2.2. $P_{\text {wap }}(\mathbb{X})$ is a Banach space equipped with the supnorm.

Now we consider asymptotically $\omega$-antiperiodic function.

Definition 2.2. A function $f \in B C(\mathbb{R}, \mathbb{X})$ is said to be asymptotically $\omega$-antiperiodic if there exist $u \in P_{\text {wap }}(\mathbb{X})$ and $h \in C_{0}\left(\mathbb{R}^{+}, \mathbb{X}\right)$, such that 


$$
f=u+h, \quad \forall t \in \mathbb{R}^{+} .
$$

$g$ and $h$ are called respectively the principal and corrective terms of $f$.

We will denote by $A P_{\text {wap }}(\mathbb{X})$, the space of all asymptotically $\omega$-antiperiodic $\mathbb{X}$ valued functions.

Remark 2.1. $A P_{\text {wap }}(\mathbb{X})$ is a Banach space equipped with the supnorm and the decomposition of an asymptotically antiperiodic is unique.

\section{Main Results}

We begin with the definition of a solution to (1).

Definition 3.1. A solution of Equation (1) on $\mathbb{R}^{+}$is a function $x(t)$ that satisfies the conditions:

$1-\mathrm{x}(\mathrm{t})$ is continuous on $\mathbb{R}^{+}$.

2-The derivative $x^{\prime}(t)$ exists at each point $t \in \mathbb{R}^{+}$, with possible exception of the points $[t] \in \mathbb{R}^{+}$where one-sided derivatives exists.

3-Equation (1) is satisfied on each interval $[n, n+1)$ with $n \in \mathbb{N}$.

Let $T(t)$ be the $C_{0}$ semigroup generated by $A$ and $x$ a solution of (1). Then the function $m$ defined by $m(s)=T(t-s) x(s)$ is differentiable for $s<t$ and we can write:

$$
\begin{aligned}
\frac{\mathrm{d} m(s)}{\mathrm{d} s}= & -A T(t-s) x(s)+T(t-s) x^{\prime}(s) \\
= & -A T(t-s) x(s)+T(t-s) A x(s) \\
& +T(t-s) A_{0} x([s])+T(t-s) g(s, x([s]))
\end{aligned}
$$

which leads to

$$
\frac{\mathrm{d} m(s)}{\mathrm{d} s}=T(t-s) A_{0} x([s])+T(t-s) g(s, x([s])) .
$$

The function $x([s])$ is a step function and $g(s, x([s]))$ is a continuous function in the intervals $[n, n+1)$, where $n \in \mathbb{N}$. Therefore, the functions $x([s])$ and $g(s, x([s]))$ are integrable over $[0, t]$ with $t \in \mathbb{R}^{+}$. Integrating both sides of (2) over $[0, t]$, yields

$$
x(t)-T(t) x(0)=\int_{0}^{t} T(t-s) A_{0} x([s]) \mathrm{d} s+\int_{0}^{t} T(t-s) g(s, x([s])) \mathrm{d} s .
$$

Therefore, we give the following

Definition 3.2. Let $T(t)$ be the $C_{0}$ semigroup generated by $A$. The function $x \in \mathcal{C}\left(\mathbb{R}^{+}, \mathbb{X}\right)$ given by

$$
x(t)=T(t) c_{0}+\int_{0}^{t} T(t-s) A_{0} x([s]) \mathrm{d} s+\int_{0}^{t} T(t-s) g(s, x([s])) \mathrm{d} s
$$

is the mild solution of the Equation (1).

Now we assume that:

(H.1) The operator A is the infinitesimal generator of an exponentially stable semigroup $(T(t))_{t \geq 0}$ such that there exist constants $M>0$ and $\delta>0$ with

$$
\|T(t)\|_{B(\mathbb{X})} \leq M \mathrm{e}^{-\delta t}, \forall t \geq 0 .
$$


The proof of the main result of this paper is based on the following two lemmas.

Lemma 3.1. Assume that (H.1) is satisfied and that $A_{0}$ is a linear bounded operator. Let $\omega \in \mathbb{N}$, we define the nonlinear operator $\Gamma_{1}$ by: for each $\phi \in A P_{\text {wap }}(\mathbb{X})$

$$
\left(\Gamma_{1} \phi\right)(t)=\int_{0}^{t} T(t-s) A_{0} \phi([s]) \mathrm{d} s .
$$

Then the operator $\Gamma_{1}$ maps $A P_{\text {कap }}(\mathbb{X})$ into itself.

Proof. Define the function $F$ by

$$
F(t)=\int_{0}^{t} T(t-s) A_{0} \phi([s]) \mathrm{d} s .
$$

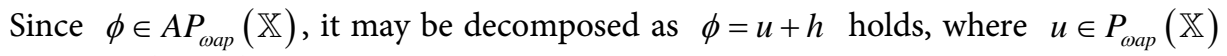
and $h \in C_{0}\left(\mathbb{R}^{+}, \mathbb{X}\right)$. We note that

$$
F(t)=G(t)+H(t), \quad t \in \mathbb{R}
$$

where

$$
G(t)=\int_{-\infty}^{t} T(t-s) A_{0} u([s]) \mathrm{d} s
$$

and

$$
H(t)=\int_{0}^{t} T(t-s) A_{0} h([s]) \mathrm{d} s-\int_{-\infty}^{0} T(t-s) A_{0} u([s]) \mathrm{d} s .
$$

We claim that $H \in C_{0}\left(\mathbb{R}^{+}, \mathbb{X}\right)$. Since $h \in C_{0}\left(\mathbb{R}^{+}, \mathbb{X}\right)$, then $\lim _{t \rightarrow+\infty} h([t])=0$. Therefore: $\forall \epsilon>0$, there exists a constant $T>0$ such that $\|h([s])\| \leq \epsilon$ for all $s \geq T$. For all $t \geq 2 T$, we have that

$$
\begin{aligned}
H(t)= & \int_{0}^{\frac{t}{2}} T(t-s) A_{0} h([s]) \mathrm{d} s+\int_{\frac{t}{2}}^{t} T(t-s) A_{0} h([s]) \mathrm{d} s \\
& -\int_{-\infty}^{0} T(t-s) A_{0} u([s]) \mathrm{d} s,
\end{aligned}
$$

from which it follows that

$$
\begin{aligned}
\|H(t)\| \leq & \int_{0}^{\frac{t}{2}} M \mathrm{e}^{-\delta(t-s)}\left\|A_{0}\right\|\|h\| \mathrm{d} s+\int_{\frac{t}{2}}^{t} M \mathrm{e}^{-\delta(t-s)}\left\|A_{0}\right\| \epsilon \mathrm{d} s \\
& +\int_{-\infty}^{0} M \mathrm{e}^{-\delta(t-s)}\left\|A_{0}\right\|\|u\| \mathrm{d} s \\
\leq & \frac{M}{\delta} \mathrm{e}^{-\delta \frac{t}{2}}\left\|A_{0}\right\| h\left\|-\frac{M}{\delta} \mathrm{e}^{-\delta t}\right\| A_{0}\|\| h\left\|+\frac{M}{\delta}\right\| A_{0} \| \epsilon \\
& -\frac{M}{\delta} \mathrm{e}^{-\delta \frac{t}{2}}\left\|A_{0}\right\| \epsilon+\frac{M}{\delta} \mathrm{e}^{-\delta t}\left\|A_{0}\right\|\|u\| .
\end{aligned}
$$

Hence, $\lim _{t \rightarrow+\infty} H(t)=0$. Since $H$ is clearly continuous, the claim is then proved. Now, we show that $G \in P_{\text {wap }}(\mathbb{X})$ :

$$
\begin{aligned}
G(t+\omega) & =\int_{-\infty}^{t+\omega} T(t+\omega-s) A_{0} u([s]) \mathrm{d} s=\int_{-\infty}^{t} T(t-s) A_{0} u([s+\omega]) \mathrm{d} s \\
& =\int_{-\infty}^{t} T(t-s) A_{0} u([s]+\omega) \mathrm{d} s=-\int_{-\infty}^{t} T(t-s) A_{0} u([s]) \mathrm{d} s .
\end{aligned}
$$

Therefore $G(t+\omega)=-G(t)$. It follows that $G \in P_{\text {wap }}(\mathbb{X})$ and $H \in C_{0}\left(\mathbb{R}^{+}, \mathbb{X}\right)$ which proves that $F \in A P_{\text {wap }}(\mathbb{X})$.

Lemma 3.2. Assume that (H.1) is satisfied and also that $\omega \in \mathbb{N}$. Let $g \in B C(\mathbb{R}, \mathbb{X})$ 


\section{be such that:}

i) $\forall(t, x) \in \mathbb{R} \times \mathbb{X}, g(t+\omega,-x)=-g(t, x)$;

ii) $\exists K>0, \forall(t, x, y) \in \mathbb{R} \times \mathbb{X} \times \mathbb{X},\|g(t, x)-g(t, y)\| \leq K\|x-y\|$.

Define the nonlinear operator $\Gamma_{2}$ by: for each $\phi \in A P_{\text {कар }}(\mathbb{X})$

$$
\left(\Gamma_{2} \phi\right)(t)=\int_{0}^{t} T(t-s) g(s, \phi([s])) \mathrm{d} s .
$$

Then the operator $\Gamma_{2}$ maps $A P_{\text {wap }}(\mathbb{X})$ into itself.

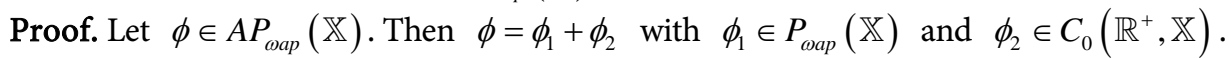
We have

$$
g(t, \phi([t]))=g\left(t, \phi_{1}([t])\right)+l(t)
$$

with $l(t)=g(t, \phi([t]))-g\left(t, \phi_{1}([t])\right)$. We have

$$
\|l(t)\| \leq K\left\|\phi_{2}([t])\right\| .
$$

Since $\lim _{t \rightarrow \infty} \phi_{2}([t])=0$, we deduce that $\lim _{t \rightarrow \infty} l(t)=0$.

We note also that $g\left(t+\omega, \phi_{1}([t+\omega])\right) \stackrel{t \rightarrow \infty}{=}-g\left(t, \phi_{1}([t])\right)$. In fact

$$
\begin{aligned}
g\left(t+\omega, \phi_{1}([t+\omega])\right) & =g\left(t+\omega, \phi_{1}([t]+\omega)\right) \\
& =g\left(t+\omega,-\phi_{1}([t])\right) \\
& =-g\left(t, \phi_{1}([t])\right) .
\end{aligned}
$$

We put

$$
F(t)=\int_{0}^{t} T(t-s) g(s, \phi([s])) \mathrm{d} s .
$$

Since the function $g$ is lipschitzian, then the function $t \rightarrow g(t, \phi([t]))$ is piecewise continuous. Therefore the function $F$ is well defined. Since $\phi=\phi_{1}+\phi_{2}$ with $\phi_{1} \in P_{\text {шар }}(\mathbb{X})$ and $\phi_{2} \in C_{0}\left(\mathbb{R}^{+}, \mathbb{X}\right)$, we observe that

$$
F(t)=G(t)+H(t)
$$

where

$$
G(t)=\int_{-\infty}^{t} T(t-s) g\left(s, \phi_{1}([s])\right) \mathrm{d} s, \quad t \in \mathbb{R}
$$

and

$$
H(t)=\int_{0}^{t} T(t-s) l(s) \mathrm{d} s-\int_{-\infty}^{0} T(t-s) g\left(s, \phi_{1}([s])\right) \mathrm{d} s, \quad t \in \mathbb{R}^{+} .
$$

The functions $G(t)$ and $l(t)$ are well defined because the function $l(t)$ and $g\left(t, \phi_{1}([t])\right)$ are continuous on $[n, n+1)$ where $n$ is an integer. Since $\lim _{t \rightarrow \infty} l(t)=0$ and $g\left(t+\omega, \phi_{1}([t+\omega])\right)=-g\left(t, \phi_{1}([t])\right)$, it follows that $G \in P_{\text {कap }}(\mathbb{X})$ and $H \in C_{0}\left(\mathbb{R}^{+}, \mathbb{X}\right)$.

Now we can state and prove the main result of this work.

Theorem 3.3. We assume that the hypothesis (H.1) is satisfied. We assume also that $\omega \in \mathbb{N}$. Let $g \in B C(\mathbb{R}, \mathbb{X})$ such that:

i) $\forall(t, x) \in \mathbb{R} \times \mathbb{X}, g(t+\omega,-x)=-g(t, x)$.

ii) $\exists K>0, \forall(t, x, y) \in \mathbb{R} \times \mathbb{X} \times \mathbb{X},\|g(t, x)-g(t, y)\| \leq K\|x-y\|$. 
Then the Equation (1) has a unique asymptotically $\omega$ antiperiodic solution if

$$
\rho:=\frac{M}{\delta}\left(\left\|A_{0}\right\|+K\right)<1
$$

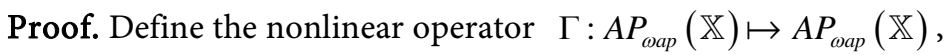

$$
(\Gamma u)(t):=L(t)+\left(\Gamma_{1} u\right)(t)+\left(\Gamma_{2} \phi\right)(t)
$$

for every $u \in A P_{\text {wap }}(\mathbb{X})$, where

$$
\begin{gathered}
L(t)=T(t) c_{0} \\
\left(\Gamma_{1} \phi\right)(t)=\int_{0}^{t} T(t-s) A_{0} \phi([s]) \mathrm{d} s
\end{gathered}
$$

and

$$
\left(\Gamma_{2} \phi\right)(t)=\int_{0}^{t} T(t-s) g(s, \phi([s])) \mathrm{d} s .
$$

Since $L(t) \in C_{0}\left(\mathbb{R}^{+}, \mathbb{X}\right)$ we have $\|L(t)\| \leq M \mathrm{e}^{-\delta t} c_{0}, \forall t \geq 0$. Then, using Lemma 3.1 and Lemma 3.2, it follows that the operator $\Gamma$ maps $A P_{\text {wap }}(\mathbb{X})$ into itself.

For every $\phi, \psi \in A P_{\text {कap }}(\mathbb{X})$,

$$
\begin{aligned}
\|\Gamma(\phi)(t)-\Gamma(\psi)(t)\| \leq & \left\|\int_{0}^{t} T(t-s) A_{0}(\phi([s])-\psi([s])) \mathrm{d} s\right\| \\
& +\left\|\int_{0}^{t} T(t-s)(g(s, \phi(s))-g(s, \psi(s))) \mathrm{d} s\right\| \\
\leq & \int_{0}^{t}\|T(t-s)\|\left\|A_{0}\right\|\|\phi([s])-\psi([s])\| \mathrm{d} s \\
& +\int_{0}^{t}\|T(t-s)\|\|(g(s, \phi(s))-g(s, \psi(s)))\| \mathrm{d} s \\
\leq & \int_{0}^{t}\|T(t-s)\|\left\|A_{0}\right\| \phi-\psi\left\|_{\infty} \mathrm{d} s+\int_{0}^{t}\right\| T(t-s)\|K\| \phi-\psi \|_{\infty} \mathrm{d} s \\
\leq & \int_{0}^{t} M \mathrm{e}^{-\delta(t-s)} \mathrm{d} s\left\|A_{0}\right\|\|\phi-\psi\|_{\infty}+\int_{0}^{t} M K \mathrm{e}^{-\delta(t-s)} \mathrm{d} s\|\phi-\psi\|_{\infty} \\
& \leq \frac{M}{\delta}\left\|A_{0}\right\|\|\phi-\psi\|_{\infty}+\frac{M}{\delta} K\|\phi-\psi\|_{\infty} \\
\leq & \frac{M}{\delta}\left(\left\|A_{0}\right\|+K\right)\|\phi-\psi\|_{\infty} .
\end{aligned}
$$

Therefore, since $\rho<1$, using the Banach fixed point Theoren we conclude that Equation (1) has a unique asymptotically $\omega$-antiperiodic solution.

\section{Application}

As an application, consider for $t \in \mathbb{R}^{+}, x \in[0, \pi]$ and $\alpha \in \mathbb{R}$, the Cauchy problem:

$$
\left\{\begin{array}{l}
\frac{\partial u}{\partial t}(t, x)=\frac{\partial^{2} u}{\partial x^{2}}(t, x)+\alpha u([t], x)+g(t, u([t], x)) ; \\
u(t, 0)=u(t, \pi)=0 .
\end{array}\right.
$$

We take $(\mathbb{X},\|\cdot\|)=\left(L^{2}([0, \pi]),\|\cdot\|_{2}\right)$ and we define the linear operator $A$ by

$$
\begin{gathered}
D(A)=\left\{v \in L^{2}([0, \pi]), v^{\prime \prime} \in L^{2}([0, \pi]), v(0)=v(\pi)=0\right\} \\
A v=v^{\prime \prime} .
\end{gathered}
$$


where the derivatives are taken in the distributional sense. Then, $A$ is the infinitesimal generator of a semigroup $T(t)$ on $L^{2}([0, \pi])$ satisfying $\|T(t)\| \leq \mathrm{e}^{-t}$ for $t \geq 0$ (see [20]). The operator $A_{0}: L^{2}([0, \pi]) \rightarrow L^{2}([0, \pi])$ defined by $A_{0}(v)=\alpha v$ is linear and bounded with $\left\|A_{0}\right\|=|\alpha|$. Therefore (3) takes the abstract form (1). Assume that the function $g \in B C(\mathbb{R}, \mathbb{X})$ satisfies the following:

i) $\forall(t, x) \in \mathbb{R} \times \mathbb{X}, g(t+\omega,-x)=-g(t, x)$,

ii) $\exists K>0, \forall(t, x, y) \in \mathbb{R} \times \mathbb{X} \times \mathbb{X},\|g(t, x)-g(t, y)\| \leq K\|x-y\|$.

Note that such a function exists. Take for instance $g(t, x)=f(t) x$ where $f$ is a $\omega$-periodic function from $\mathbb{R}$ into $\mathbb{R}$. Then we have

$$
g(t+\omega,-x)=f(t)(-x)=-g(t, x)
$$

and

$$
\|g(t, x)-g(t, y)\|=\|f(t)(x-y)\| \leq\|f\|_{\infty}\|x-y\| .
$$

Theorem 4.1. We assume that $\omega \in \mathbb{N}$. Then System (3) has a unique asymptotically $\omega$-antiperiodic if $|\alpha|+K<1$.

Proof. We have $M=1, \delta=1,\left\|A_{0}\right\|=|\alpha|$ and we apply Theorem 3.3.

\section{References}

[1] Shah, S.M. and Wiener, J. (1983) Advanced Differential Equations With Piecewise Constant Argument Deviations. International Journal of Mathematics and Mathematical Sciences, 6, 671-703. http://dx.doi.org/10.1155/S0161171283000599

[2] Van Minh, N. and Tat Dat, N. (2007) On the Almost Automorphy of Bounded Solutions of Differential Equations with Piecewise Constant Argument. Journal of Mathematical Analysis and Application, 326, 165-178. http://dx.doi.org/10.1016/j.jmaa.2006.02.079

[3] Wiener, J. (1999) A Second-Order Delay Differential Equation with Multiple Periodic Solutions. Journal of Mathematical Analysis and Application, 229, 6596-676. http://dx.doi.org/10.1006/jmaa.1998.6196

[4] Wiener, J. and Debnath, L. (1997) Boundary Value Problems for the Diffusion Equation with Piecewise Continuous Time Delay. International Journal of Mathematics and Mathematical Sciences, 20, 187-195. http://dx.doi.org/10.1155/S0161171297000239

[5] Wiener, J. and Debnath, L. (1995) A Survey of Partial Differential Equations with Piecewise Continuous Arguments. International Journal of Mathematics and Mathematical Sciences, 18, 209-228. http://dx.doi.org/10.1155/S0161171295000275

[6] Wiener, J. and Lakshmikantham, V. (1999) Excitability of a Second-Order Delay Differential Equation. Nonlinear Analysis, 38, 1-11. http://dx.doi.org/10.1016/S0362-546X(98)00245-4

[7] Wiener, J. (1993) Generalized Solutions of Functional Differential Equations. World Scientific, Singapore, New Jersey, London, Hong Kong.

[8] Henríquez, H.R., Pierre, M. and Táboas, P. (2008) On S-Asymptotically $\omega$-Periodic Function on Banach Spaces and Applications. Journal of Mathematical Analysis and Applications, 343, 1119-1130. http://dx.doi.org/10.1016/j.jmaa.2008.02.023

[9] Henríquez, H.R., Pierre, M. and Táboas, P. (2008) Existence of S-Asymptotically $\omega$-Periodic Solutions for Abstract Neutral Equations. Bulletin of the Australian Mathematical Society, 78, 365-382. http://dx.doi.org/10.1017/S0004972708000713

[10] Liu, Y. and Liu, X. (2013) New Existence Results of Anti-Periodic Solutions of Nonlinear 
Impulsive Functional Differential Equations. Mathematica Bohemica, 138, 337-360.

[11] Al-Islam, N.S., Alsulami, S.M. and Diagana, T. (2012) Existence of Weighted Pseudo Anti-Periodic Solutions to Some Non-Autonomous Differential Equations. Applied Mathematics and Computation, 218, 6536-6648. http://dx.doi.org/10.1016/j.amc.2011.12.026

[12] Alvarez, E., Lizama, C. and Ponce, R. (2015) Weighted Pseudo Antiperiodic Solutions for Fractional Integro-Differential Equations in Banach Spaces. Applied Mathematics and Computation, 259, 164-172. http://dx.doi.org/10.1016/j.amc.2015.02.047

[13] Chen, H.L. (1996) Antiperiodic Functions. Journal of Computational Mathematics, 14, 3239.

[14] Chen, Y.Q. (2006) Anti-Periodic Solutions for Semilinear Evolution Equations. Journal of Mathematical Analysis and Applications, 315, 337-348. http://dx.doi.org/10.1016/j.jmaa.2005.08.001

[15] Freire, J.G., Cabeza, C., Marti, C., Pöschel, T. and Gallas, J.A.C. (2013) Antiperiodic Oscillations. Scientific Reports, 3, Article Number: 1958. http://dx.doi.org/10.1038/srep01958

[16] Haraux, A. (1989) Anti-Periodic Solutions of Some Nonlinear Evolution Equations. Manuscripta Mathematica, 63, 479-505. http://dx.doi.org/10.1007/BF01171760

[17] Okoshi, H. (1990) On the Existence of Antiperiodic Solutions to a Nonlinear Evolution Equation Associated with Odd Subdifferential Operators. Journal of Functional Analysis, 91, 246-258. http://dx.doi.org/10.1016/0022-1236(90)90143-9

[18] N'Guérékata, H. and Valmorin, V. (2012) Antiperiodic Solutions of Semilinear Integrodifferential Equations in Banach Spaces. Applied Mathematics and Computation, 218, 11118 11124. http://dx.doi.org/10.1016/j.amc.2012.05.005

[19] Mophou, G., N'Guérékata, G. and Valmorin, V. (2013) Asymptotic Behavior of Mild Solutions of Some Fractional Functional Integro-Differential Equations. African Diaspora Journal of Mathematics, 16, 70-81.

[20] Diagana, T. and N'Guérékata, G.M. (2007) Almost Automorphic Solutions to Some Classes of Partial Evolution Equations. Applied Mathematics Letters, 20, 462-466. http://dx.doi.org/10.1016/j.aml.2006.05.015

\section{Submit or recommend next manuscript to SCIRP and we will provide best service for you:}

Accepting pre-submission inquiries through Email, Facebook, LinkedIn, Twitter, etc. A wide selection of journals (inclusive of 9 subjects, more than 200 journals)

Providing 24-hour high-quality service

User-friendly online submission system

Fair and swift peer-review system

Efficient typesetting and proofreading procedure

Display of the result of downloads and visits, as well as the number of cited articles Maximum dissemination of your research work

Submit your manuscript at: http://papersubmission.scirp.org/ 